(5) Follow-up: out-patient appointment arranged.

Hensol Hospital, Pontyclun, Mid Glamorgan, and Llwyneryr Hospital Morriston, Swansea

\section{Benzodiazepines and ECT}

\section{DEAR SIRS}

While it is accepted that benzodiazepines have anticonvulsant activity, most psychiatrists would not prescribe them and administer ECT at the same time in the belief that seizure is the necessary requirement for the patient to get better, the reality of the interactions seem to be more complicated than that.

Firstly, it is known that there are depressed patients who seem to have adequate seizures during ECT treatment, but still remain equally depressed. Secondly, there are patients who are on small doses of benzodiazepines, get ECT, have seizures and improve. Thirdly, there are patients who are in the process of withdrawal from benzodiazepines and develop depression as a result (Lader et al, 1981) that seems to be difficult to treat with antidepressants and most likely ECT too. During withdrawal, patients experience among many other symptoms those of depression, and major convulsions or temporal lobe seizures sometimes occur on abrupt withdrawal (Ashton, 1986). As these symptoms can occur together I would suggest that for several weeks following the withdrawal of benzodiazepines patients would show altered responses to ECT and antidepressants. My concern is that clinicians might misunderstand this to indicate that in future ECT should not be given on the grounds that: "s/he does not respond to ECT". It would be interesting to hear from other psychiatrists if they have found altered response to ECT as a result of benzodiazepine withdrawal.

The London Hospital

BisA HAEGER

Whitechapel Road

London E1 1BB

\section{References}

Ashton, H. (1986) Adverse effects of prolonged benzodiazepine use. Adverse Drug Reaction Bulletin, 118, 440-443.

LADER, M. H. \& Petursson, H. (1981) Benzodiazepine derivatives - side effects and dangers. Biological Psychiatry, 16, 1195-1221.

\section{The double negatives and the Mental Health Review Tribunal}

DeAR SIRS

We would like to express through your correspondence columns some difficulties we have experienced in conveying the reports of the Mental Health
Review Tribunal to our patients who had appealed against their detention under the Mental Health Act 1983. Here are two examples of the Tribunal's decision on two patients detained under Section 2 (extracted from form 7);

(1) "The Tribunal is not satisfied that .... is not now suffering from mental disorder of a nature and degree which warrants his/her detention in a hospital for assessment. The Tribunal is not satisfied that it is not necessary in the interests of the patient's health and safety that she should be detained...."

(2) "They are not satisfied that he/she is not suffering from mental disorder... They are also not satisfied that his/her detention as aforesaid is not justified in the interests of his/her own health...."

In both cases the Tribunal accepted the medical and social worker's opinion and since the Tribunal had no objection to the reasons for their decision being "fully disclosed" to the patient they were conveyed to the patients and in both cases the patients insisted on seeing the reports for themselves. After reading the report both patients refused to believe that they had lost their appeal and had great difficulty in interpreting the double negatives. One patient's appeal against her subsequent detention under Section 3 was, we believe, related to her inability to understand the Tribunal's report on her initial appeal against Section 2.

Until the 17th century the use of double or multiple negatives was permitted in educated English as a form of emphasis (International English Usage, Croom Helm): "Nor go neither; but you'll lie like dogs, and yet say nothing neither" (Shakespeare, The Tempest, Act 3, Scene 2). This form is now only used in dialects, e.g. "He didn't say nothing".

The use of double negatives is still legitimate in educated English when they combine to express a positive (Longman Guide to English Usage). In the example "a not unhappy choice" or "not infrequently" the word "not" negates the negative word to produce a "weak positive". While in "You cannot not admire her pluck" and "None of us have no friends" a "strong positive" effect is produced. The above two examples from the Tribunal reports are similar to the later examples of "strong positive" and yet they caused consternation and confusion in our patients.

The capacity to understand such linguistic points will depend, of course, upon the level of sophistication that the patient possesses. Only a few patients are highly literate and though the disorder of mood and thinking can cause problems in understanding the written word, we believe that our patients were stymied by the style of the language used. There is no denying(!) the fact that the use of multiple negatives makes the sentence difficult to understand even for normal people, and the less said the better for terms like 'aforesaid', 'heretofore' and 'notwithstanding'. 